\title{
Tiña pedis en estudiantes de la Universidad del Valle, Cali, Colombia
}

\author{
María Inés Alvarez , Luz Angela González de Polanía²
}

\begin{abstract}
Resumen
Se analizaron 333 estudiantes de la Universidad del Valle en Cali, Colombia, con el fin de determinar la frecuencia de afección micótica en el cuarto espacio interdigital del pie, la cual se estableció por examen directo, culcivc o ambos.
\end{abstract}

La prevalencia global de infección micótica fue de 15,6\%, en tanto que para tiña pedis fue de 10,5\%. El mayor número de casos se halló en estudiantes entre 21 y 25 años; se encontró que la prevalencia aumentaba con relación a la edad. No se hallaron diferencias significativas en la distribución por sexos; tampoco se encontró asociación entre el grupo sanguíneo y la práctica deportiva entre los individuos con o sin infección micótica. Los agentes aislados fueron dermatofitos y no dermatofitos; los primeros causaron 35 casos, los segundos 4 y en 13 no se logró aislar hongos. El dermatofito más aislado fue Trichophyton mentagrophytes $(71,4 \%)$, seguido en menor proporción por Trichophyton rubrum y Epidermophyton floccosum. Además, se encontró que, de 52 individuos positivos, $17(32,7 \%)$ no presentaban lesiones, convirtiéndose, por tanto, en portadores sanos. Se destaca la importancia de mantener unas adecuadas condiciones higiénicas de los pies para evitar la tiña pedis.

\section{Tinea pedis amongst students from Universidad de Valle, Colombia.}

In order to determine mycotic infection of the fourth pedal interdigital space, 333 students from Universidad del Valle, in Cali, Colombia were investigated. The infection was confirmed by direct examination, culture or both.

The global prevalence of mycotic infection was $15.6 \%$, whilst for tinea pedis it was $10.5 \%$. Infection occurred most frequently in students aged 21-25 years; it was found that prevalence increased with age.

There was no correlation with sex, blood group, sport practice and the presence of mycotic infection.

The isolated agents were dermatophytes and non-dermatophytes, the first caused 35 cases, the second one 4 and in 13 cases no fungi were isolated. The dermatophyte most frequently isolated was Trichophyton mentagrophytes $(71.4 \%)$, Trichophyton rubrum, followed by Epidermophyton floccosum. Also, it was found that of 52 positive individuals, $17(32.7 \%)$ did not show lesions, being therefore healthy carriers. It is important to highlight the development of appropriate hygienic foot conditions in order to avoid tinea pedis.

\footnotetext{
${ }^{1}$ Departamento de Microbiología, Facultad de Salud, Universidad del Valle, Cali, Colombia.

${ }^{2}$ Departamento de Laboratorio Clínico, Facultad de Salud, Universidad del Valle, Cali, Colombia.

Recibido para su publicación: 19 de agosto de 1998 - Aceptado para su publicación: 22 de diciembre de 1998
} 
La tiña pedis, entidad cutánea causada por hongos dermatofitos, constituye un problema de salud pública importante en muchos países latinoamericanos, donde el clima húmedo y cálido permite la existencia de sus agentes etiológicos. Es la dermatomicosis más común del hombre y, como su nombre, lo indica invade la piel de los pies (1-4). El cuarto espacio interdigital es el sitio más afectado (5, 6); la humedad y el calor que alberga esta área, proporcionan un ambiente que estimula el desarrollo de los hongos (1). Se manifiesta con grados variables de descamación, enrojecimiento, fisuras y maceración $(1,3)$.

El índice de afección para esta patología en la población oscila entre 30 y $70 \%$; la mayoría son casos ocultos o subclínicos (1). La baja prevalencia $(4 \%)$ de esta afección en indígenas colombianos se atribuye a la ausencia de calzado (7), mientras que las encuestas de otras poblaciones no indígenas informan prevalencias de 22 a $75 \%(2,5,8)$. La tiña pedis se considera un padecimiento urbano, donde las personas que usan zapatos proveen las condiciones necesarias para su desarrollo (4).

El presente trabajo se realizó con el propósito de conocer la frecuencia de tiña pedis interdigital en los estudiantes de la Facultad de Salud de la Universidad del Valle y establecer su asociación con la edad, el sexo, la presencia de lesiones, el agente etiológico, la práctica deportiva y el grupo sanguíneo.

\section{Materiales y métodos}

El estudio se realizó entre 1991-1993 en 333 estudiantes de los programas académicos de medicina, enfermería y bacteriología de la Facultad de Salud de la Universidad del Valle, Cali, Colombia. A cada uno se le practicó un raspado con bisturí del espacio interdigital entre el cuarto y el quinto artejo del pie. Se llevó a cabo un examen directo con $\mathrm{KOH}$ al $20 \%$ más tinta Parker azul-negra y un cultivo en agar selectivo para hongos patógenos (Merck). Los cultivos se incubaron por 2 semanas a temperatura ambiente y los hongos se identificaron por la apariencia macroscópica y sus características microscópicas (9). Además, en el caso de las levaduras, se determinó la hidrólisis de la urea, la producción de tubo germinal y la actividad de la ureasa (1).

De cada estudiante se obtuvieron los datos de edad, sexo, grupo sanguíneo, práctica deportiva y presencia de lesiones.

Los resultados se incluyeron en una base de datos y se realizó el análisis mediante el paquete estadístico Epi-Info, versión 6.0, del CDC; se asumió un nivel de significancia de $95 \%$. Además, se aplicó la prueba chi cuadrado, donde el valor $p<0,05$ se consideró estadísticamente significativo.

\section{Resultados}

De un total de 333 individuos, 216 (64,9\%) correspondieron a estudiantes de medicina, $62(18,6 \%)$ de bacteriología y $55(15,5 \%)$ de enfermería; 191 (57,3\%) eran mujeres y 142 $(42,6 \%)$ hombres. Se confirmó la afección micótica por examen directo, cultivo o ambos en 52 sujetos. En el cuadro 1 se observa que, en este estudio, la prevalencia global de infección micótica fue de $15,6 \%$, en tanto que para tiña pedis fue de $10,5 \%$ y de $1,2 \%$ para dermatomicosis causada por hongos no dermatofitos.

De los 52 estudiantes positivos, $46,2 \%$ pertenecía al sexo femenino y $53,8 \%$ al masculino; no hubo diferencia significativa en la distribución por sexo.

El promedio de edad del total de estudiantes fue de 21,5 , con rangos de 17 a 40 años; el cuadro 2 muestra la relación entre grupo de edad y la presencia de infección micótica. Se observa que la mayoría de estudiantes eran menores de 26 años y que el número mayor de casos estuvo entre 21-25 años, duplicando el número presente en el rango entre 17-20. Se encontró entre estos dos grupos una diferencia estadísticamente significativa en la presentación de infección $\left(\mathrm{Chi}^{2}=8,48, \mathrm{p}=0,01\right.$ con $1 \mathrm{gl})$. El análisis de tendencia lineal mostró que la edad fue un factor asociado con la presencia de la micosis $(p<0,006)$. 
Cuadro 1. Prevalencia de infección micótica según año de estudio (prevalencia $\times 100$ ).

\begin{tabular}{cccccr} 
Año & $\begin{array}{c}\text { Dermatofitos } \\
\text { (Tiña pedis) }\end{array}$ & $\begin{array}{c}\text { No dermatofitos } \\
\text { (Dermatomicosis) }\end{array}$ & $\begin{array}{c}\text { Diagnosticados } \\
\text { solo por ED* }\end{array}$ & $\begin{array}{c}\text { Total casos } \\
\text { infección micótica }\end{array}$ & $\begin{array}{c}\text { Total individuos } \\
\text { estudiados }\end{array}$ \\
\hline 1991 & $10(10,8)$ & $1(1,0)$ & $9(9,7)$ & $20(21,5)$ & 93 \\
1992 & $12(11,4)$ & 0 & $2(1,9)$ & $14(13,3)$ & 105 \\
1993 & $13(9,6)$ & $3(2,2)$ & $2(1,5)$ & $18(13,3)$ & 135 \\
\hline Total & $\mathbf{3 5 ( 1 0 , 5 )}$ & $\mathbf{4 ( 1 , 2 )}$ & $\mathbf{1 3 ( 3 , 9 )}$ & $\mathbf{5 2 ( 1 5 , 6 )}$ & $\mathbf{3 3 3}$ \\
\hline
\end{tabular}

* ED: examen directo

Cuadro 2. Análisis de tendencia lineal de la distribución de los estudiantes por edad.

\begin{tabular}{llc}
\hline Edad & Casos positivos & $\begin{array}{c}\text { Total } \\
\text { (Prevalencia } \mathbf{~ 1 0 0 ) ~}\end{array}$ \\
\hline $16-20$ & 14 & $148(9,4)$ \\
$21-25$ & 31 & $156(19,8)$ \\
26 o más & 7 & $29(24)$ \\
\hline Total & $\mathbf{5 2}$ & $\mathbf{3 3 3}$ \\
\hline
\end{tabular}

$\mathrm{Chi}^{2}$ para tendencia lineal $=8,48, \mathrm{p}<0,006$

De los 52 sujetos con infección micótica, se logró el aislamiento de hongos en 39; de ellos, $19(48,7 \%)$ fueron positivos tanto en el examen directo como en el cultivo, mientras que en $20 \mathrm{el}$ diagnóstico se hizo por el aislamiento del hongo en cultivo (cuadro 3). En los 13 casos restantes, sólo fue posible hacer el diagnóstico por observación de las estructuras micóticas en el examen directo, puesto que los cultivos fueron negativos.

Los agentes aislados se identificaron como dermatofitos y no dermatofitos. Para relacionar estos últimos como causantes de infección, se tuvieron en cuenta los siguientes criterios, examen directo positivo y crecimiento puro del hongo en los sitios de siembra. El cuadro 3 muestra que, de 39 aislamientos, 35 (89,7\%) correspondieron a dermatofitos y el resto a otros no dermatofitos. Entre los primeros, la especie más común fue Trichophyton mentagrophytes $(71,4 \%)$ y, entre los no dermatofitos, Candida albicans.

De los 333 estudiantes, 52 presentaron algún procedimiento diagnóstico positivo, distribuidos en $35(67,3 \%)$ con lesión y $17(32,7 \%)$ sin lesión. Los 281 restantes en los que se obtuvieron procedimientos diagnósticos negativos, 17 casos (6\%) presentaban lesión y 264 no la presentaban. El análisis de estos resultados mostró diferencias significativas $\left(\mathrm{Chi}^{2}\right.$ con corrección de Yates $=120,35, p<0,0001$ con 1 grado de libertad).

Cuando se analizó la micosis con relación a la práctica o no de deportes, se encontró que de 172 estudiantes que practicaban algún deporte, 29 presentaron infección micótica; mientras que, entre 161 que no la realizaban, se hallaron 23 individuos con la afección, sin mostrar diferencia estadística significativa $\left(\mathrm{Chi}^{2} \mathrm{con}\right.$ corrección de Yates $=0,64, p=0,4249$ con 1 grado de libertad).

Cuadro 3. Aislamiento de hongos en el cuarto espacio interdigital de 39 estudiantes.

\begin{tabular}{lccc}
\hline $\begin{array}{c}\text { Agentes etiológicos } \\
\text { Dermatofitos }\end{array}$ & $\begin{array}{c}\text { ED (+) y } \\
\text { cultivo (+) }\end{array}$ & $\begin{array}{c}\text { ED (-) y } \\
\text { cultivo (+) }\end{array}$ & Total \\
\hline T. mentagrophytes & 8 & 17 & 25 \\
T. rubrum & 4 & 1 & 5 \\
E. floccosum & 3 & 1 & 4 \\
T. mentagrophytes + T. rubrum & 1 & 0 & 35 \\
Subtotal dermatofitos & 16 & 19 & 2 \\
No dermatofitos & 1 & 1 & 1 \\
C. albicans & 1 & 0 & 1 \\
Candida sp. & 1 & 0 & 4 \\
N. mangiferae & 3 & 1 & 39 \\
Subtotal no dermatofitos & $\mathbf{1 9}$ & $\mathbf{2 0}$ & \\
\hline Total & & & \\
\hline
\end{tabular}


En cuanto a la distribución por grupos sanguíneos, no se encontraron diferencias significativas, observándose que tanto en el grupo afectado como en el negativo el predominio del grupo sanguíneo fue el $\mathrm{O}$ positivo con 57 y $55 \%$, respectivamente, seguido del A positivo con 17 y $24 \%$.

\section{Discusión}

En el presente trabajo se determinó una prevalencia de $10,5 \%$ para dermatofitos (tiña pedis). Al comparar la prevalencia de tiña pedis con la obtenida en otras poblaciones de estudiantes, se encontró que coincide con un estudio realizado en la Universidad de Otago, Nueva Zelanda, donde se informa una prevalencia de $10,6 \%$ (10) y es menor que la obtenida en un estudio realizado en Cali por Ramírez et al. en estudiantes de sexto año de bachillerato donde se informa una prevalencia de $22 \%$ (2); es posible que la menor prevalencia detectada en los estudiantes de la universidad esté relacionada con el mayor conocimiento de las medidas preventivas que tienen los estudiantes de una facultad de salud.

Se determinó que $89,7 \%$ de las infecciones fueron causadas por hongos dermatofitos y $10,3 \%$ por otro tipo. En 13 casos se obtuvo un examen directo positivo, pero no se logró el aislamiento del agente etiológico; varias pueden ser las causas, entre ellas, infecciones incipientes, poco material clínico obtenido o la sensibilidad de hongos no dermatofitos a la cicloheximida, componente incorporado en el medio utilizado.

Debe destacarse el hallazgo de cuatro no dermatofitos que correspondieron, dos a $C$. albicans, uno a Candida sp. y otro a Nattrassia mangiferae. Vale la pena resaltar que cuando se aislan dermatofitos o C. albicans son considerados sin duda los agentes etiológicos del proceso, por ser verdaderos patógenos; sin embargo, otros hongos que incluyen varias especies de Candida, son de dudosa patogenicidad a menos que se cumpla una serie de criterios, como son la comprobación repetida por examen directo y cultivo (11). En este caso, Candida sp. y N. mangiferae se incluyeron como agentes etiológicos aunque su aislamiento no se repitió, por haberse observado estructuras micóticas en el examen directo que indicaban la presencia de una levadura y moho, respectivamente, además, de encontrarse en los sitios de siembra en cantidad abundante en los cultivos. Es importante tener presente a los hongos considerados como saprófitos como posibles causantes de infección $(4,12-14)$ y emplear medios que no contengan cicloheximida para el aislamiento de este tipo de hongos.

Los informes de la literatura colombiana señalan a $T$. mentagrophytes como el principal agente etiológico de la tiña pedis en Colombia $(2,4,11$, 15-17). En el cuadro 2, se puede apreciar que $71,4 \%$ de las infecciones por dermatofitos fueron causadas por este hongo, lo cual muestra la concordancia de este resultado con los informes mencionados.

En este estudio, T. rubrum ocupa el segundo puesto dentro de los dermatofitos causantes de tiña pedis interdigital con 5 casos $(14,3 \%)$ mientras que, a nivel mundial, este hongo es el agente predominante $(1,18-24)$.

Entre los 281 estudiantes negativos, 6\% presentó lesiones interdigitales sin que hubiera infección micótica; se sabe que existen otras causas de esta afección como alergias, dermatitis por contacto, piodermitis y eczemas (1)

Mercantini et al. señalan la edad como un factor importante para el establecimiento de las dermatofitosis (19), encontrando que a medida que se avanza en edad se halla una mayor frecuencia. En el presente trabajo, se observó un mayor número de casos entre los estudiantes con edades entre 21 y 25 años que entre los de 16 a 20; el análisis de tendencia lineal demostró que éstas aumentaban con la edad.

Respecto al sexo, se halló mayor número de casos en el sexo masculino a pesar de que el número de mujeres estudiadas fue superior; estos resultados son similares a otros estudios que muestran que la mayor frecuencia de tiña pedis es en hombres $(2,15,18,21)$. 
Con relación al grupo sanguíneo, no se encontraron diferencias significativas, aunque Nielsen halló evidencias que sugerían asociación entre este factor y la tiña pedis (25).

En este estudio se considera que los estudiantes con lesiones y procedimientos positivos padecen la enfermedad. Aquellos con el examen directo o el cultivo positivos, a pesar de no presentar lesiones, son portadores sanos del agente causal y constituyen un foco de dispersión del hongo.

La tiña pedis se adquiere por contacto con escamas infectadas y, a partir de éstas, puede continuar e involucrar las uñas de los pies y la piel glabra (1). Esta situación debe estimular el emprender campañas educativas que concienticen a los individuos con esta entidad para que eviten caminar descalzos cerca de las piscinas, las duchas o los lugares públicos y, de esta manera, no exponer a la comunidad. Además, se recomienda tomar una serie de medidas preventivas como mejorar la higiene de los pies (lavado regular, un perfecto secado y aplicación de polvos), evitar la excesiva humedad y oclusión usando sandalias o calzado bien ventilado y no compartir toallas, medias ni zapatos (5).

\section{Referencias}

1. Rippon JW. Medical mycology. The pathogenic fungi and the pathogenic actinomycetes. 3rd. ed. Philadelphia: W. B. Saunders Co; 1988.p.218-20.

2. Ramírez M, Hoyos MP, Lorza AM, Greer DL. Tiña pedis en alumnos de sexto año de bachillerato en cuatro colegios de Cali. Acta Med Valle 1978;9:7680 .

3. Restrepo A. Dermatomicosis. En: Restrepo A, Robledo J, Bedoya V, Restrepo $M$, Botero D, Leyderman E, Betancur J, Gómez C, Vélez L, editores. Fundamentos de medicina. Enfermedades infecciosas. $5^{\mathrm{a}}$. ed. Medellín: Corporación para Investigaciones biológicas; 1996.

4. Escobar ML, Ortega MC, Muñoz V, Montoya F, Martínez D, Santamaría L, Vargas ME. Dermatomicosis en deportistas. latreia 1992;2: 23-7.

5. Greer DL, Ayabaca JC, Quiceno M, Bonilla A. Epidemiological studies of dermatophytosis in Colombia. Fifth International Conference on
Mycology. PAHO Scient. Publ. \#396. Washington, D. C.: WHO; 1980.p.2730.

6. Weitzman Y, Summerbell R. The dermatophytes. Clin Microbiol Rev 1995;240-59.

7. Greer DL, Ayabaca JC, Quiceno M. Factores que afectan la prevalencia de dermatomicosis en dos localidades indígenas de Colombia. Colombia Med 1981;12:54-60.

8. Flórez E, Gaviria B, Gómez BL, González A, Vélez J, Calle C, Gutiérrez CG, Franco Q, García AH, Gómez LF. Aislamiento de agentes micóticos en una piscina de la ciudad de Medellín, Colombia. Antioquia Med 1976;26:427-36.

9. Rebell, Taplin D. Dermatophytes: their reconigtion and identificacion. Coral Gables: University of Miami Press; 1972.

10. Marples M, DiMenna ME. A survey of the incidence of interdigital fungus infections in a group of students from the University of Otago. Med $\mathrm{J}$ Aus 1949;2:156-61.

11. Escobar ML, Vélez H, Santamaría L, Guzmán G, Restrepo B, Ceballos G, Díaz F. Dermatomicosis y onicomicosis en estudiantes de una escuela de policía. latreia 1989;2:29-36.

12. Leyden J, Kligman AM. Interdigital athlete's foot. Arch Dermatol 1978;114:1466-72.

13. Summerbell RC, Kane J, Krajden S. Onychomycosis, tinea pedis and tinea manus caused by non-dermatophytic filamentous fungi. Mycoses 1989;32:609-19.

14. Vélez H. Infecciones interdigitales por hongos no dermatofíticos. latreia;1990;3:30-2.

15. Escobar ML, Ortega MC, Muñoz V, Vargas ME. Tiña pedis y otras entidades dermatológicas en un grupo de niños con retardo mental. Iatreia 1991;4: 113.

16. Polanía LA de, Alvarez MI. Dermatofitosis. Hospital Universitario del Valle, Cali, Colombia, 1978-1988. Colombia Med 1991;22:14-8.

17. Duque $\mathbf{C}$, Giraldo E, López $\mathbf{O}$, Arcila A. Tiña pedis y otras micosis cutáneas en soldados de cuatro batallones de Medellín. Biomédica 1997;17(Supl. 2):219-20.

18. Sberna F, Farella V, Geti V, Taviti F, Agostini G, Vannini P, Knopfel B, Difonzo EM. Epidemiology of the dermatophytoses in the Florence area of Italy, 1985-1990. Mycopathologia 1993;122:153-62.

19. Mercantini R, Moretto D, Palamara G, Mercantini P, Marsella R. Epidemiology of dermatophytoses observed in Rome, Italy, between 1985 and 1993. Mycoses 1995;38:415-9.

20. Buchavald J, Simaljaková M. The occurrence of dermatophytes in Slovakia. Mycoses 1995;38:159-61. 
21. Lehenkari E, Silvennoinen-Kassinen S. Dermatophytes in northern Finland in 1982-90. Mycoses 1995;38:411-4.

22. Noguchi H, Hiruma $M$, Kawada A, Ishibashi A, Kono S. Tinea pedis in members of the Japanese self-defence forces: relationships of its prevalence and its severity with length of military service and width of interdigital spaces. Mycoses 1995;38:495-9.

23. Korting HC, Blecher P, Stallmannn D, Hamm G. Dermatophytes on the feet of HIV-infected patients: frequency, species distribution, localization and antimicrobial susceptibility. Mycoses 1993;36:271-4.

24. Devliotou-Panagiotidou D, Koussidou-Eremondi T, Badillet G. Dermatophytosis in northern Greece during the decade 1981-1990. Mycoses 1995;38: $151-7$.

25. Nielsen PG. Immunological aspects of dermatophyte infections in hereditary palmo-plantar keratoderma. Acta Derm Venerol 1984;64:296-301. 